\title{
Automatic Analysis of the Left Ventricle in the Time Sequences of 3D Echo-Cardiographic Images
}

\author{
O. Gerard, M. Fradkin, A. Collet Billon, M. Jacob, J.-M. Rouet, and S. Makram-Ebeid \\ LEP - Philips Research \\ 22 av. Descartes, F-94453 Limeil-Brevannes, France \\ \{olivier.Gerard|Maxim.Fradkin\}@philips.com
}

\section{Introduction}

The 3D ultrasound imagery becomes more and more attractive for cardiac studies due to its simplicity, its improved reproducibility, and better precision, as compared to standard 2D echographic exams. However, automatic tools are needed to fully and efficiently analyze the large amounts of data obtained. In this paper, we present an automatic tool, aimed at the quantitative analysis of heart motion, based on the segmentation of the endo-cardium of the left ventricle. Using our method, quantitative measurements on volumes such as stroke and ejection-fraction $(\mathrm{EF})$ are readily available (without needing any geometrical assumption), as well as regional wall motion parameters.

\section{Method}

The method is based on 3D model-based segmentation of time sequences images. Our $3 \mathrm{D}$ geometric model is described by a 2-simplex mesh. A 2 -simplex mesh is a discrete non-parametric surface representation, defined by a set of 3 -connected vertices. A great variability in possible cell shapes allows both very accurate and compact surface representation. The model deformation is expressed in displacement of mesh vertices, governed by two forces: external, attracting the model to the object boundaries, and internal, regularizing its surface (see [1]). Due to the limited scope of this paper, the reader may refer to [2] for details. The 3D+t segmentation procedure starts with the deformation of an initial model for the first image of the cardiac cycle. This initial model of the studied chamber is manually adjusted (with just 4 mouse clicks) to the data. Then the deformation procedure is repeated for the subsequent images, using the model derived from the previous image in the cardiac cycle as initial model. Another approach [3] , in contrast with ours, uses a topological correspondence between the successive models. However, this topological correspondence does not necessarily imply the tracking of the same anatomical points of the heart, thus leading to an over-regularized final 4D model. Our approach, although quite simple, is very fast and completely automatic. Having the sequence of 3D models, one can easily calculate different heart parameters, such as the blood volume, stroke volume, and EF. Moreover, we can quantify the local wall motion of the heart. 


\section{Results}

For the validation of the proposed method, we have used 3D ultrasound data sets acquired using a rotating probe gated on the ECG (ATL, Seattle). The volume measurements have been validated using a dynamically pumped balloon-in-balloon phantom (D. J. Sahn, OSHU, Portland, OR), showing excellent accuracy (error less than the setup precision) and a very good robustness with respect to initialization and parameter setting (with standard deviation $<1.2 \mathrm{ml}$ ).

The method is able to quantify the radial motion of the LV and to represent in color this information. It has been successfully tested on the LV for both trans-esophageal and trans-thoracic 3D acquisitions as well as on other cardiac chambers (RV, atrium). Fig. 1 shows the segmentation results for two phases of the cardiac cycle of a 3D transesophageal LV dataset of a patient suffering from a large mitral regurgitation. Complete volume calculation (and motion analysis) can be performed in less than 3 minutes on a standard PC. Clinical validations are under way to assess the accuracy of the whole process, so as to be ready when real-time $3 \mathrm{D}$ echocardiography becomes clinical routine procedure.

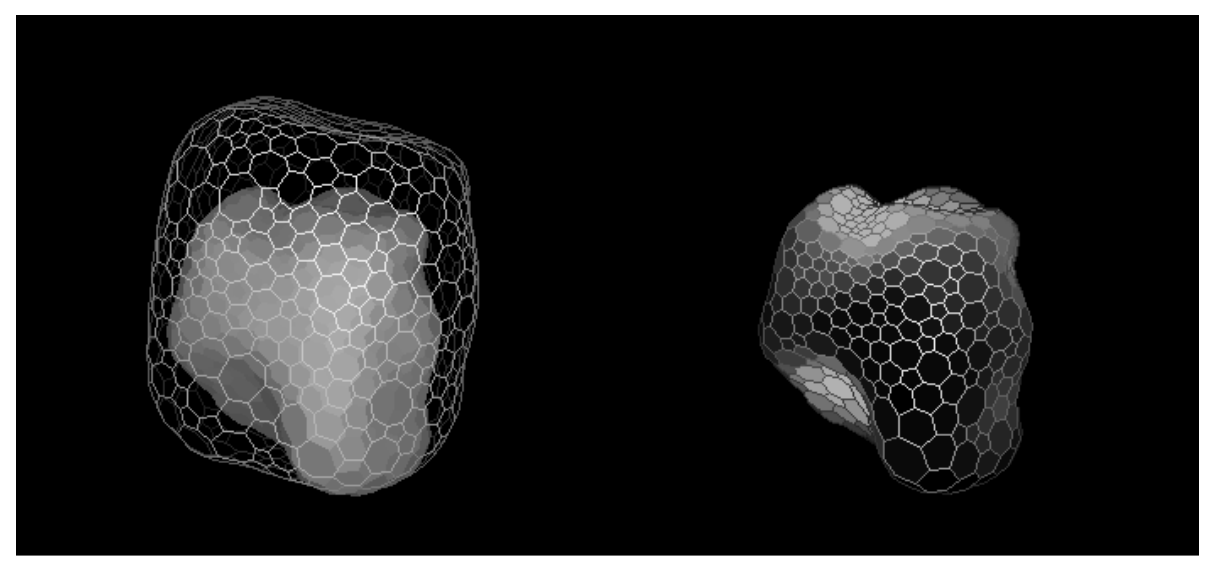

Fig. 1. Resulting model: superimposition of two active models, corresponding to endsystolic and end-diastolic phases (left); wall motion magnitude of systolic phase coding with gray levels (right)

\section{References}

1. Kass, M., Witkin, A., Terzopoulos, D.: Snakes: Active contour models. Int. J. Comp. Vision 4-1 (1988) 321-331

2. Delingette, H.: General Object Reconstruction Based on Simplex Meshes. Int. J. Comp. Vision 32-2 (1999) 111-146

3. Montagnat, J., Delingette, H.: Space and Time Shape constrained Deformable Surfaces for 4D Medical Image Segmentation. In: Delp, S., et al. (eds.): MICCAI-2000. Lecture Notes in Computer Science, Vol. 1935. Springer-Verlag, (2000) 196-205 